\title{
A simple model for cavitation with non-condensable gases
}

\author{
Mathieu Bachmann, Siegfried Müller \\ Institut für Geometrie und Praktische Mathematik, RWTH Aachen \\ 52056 Aachen, Germany. \\ E-mail:bachmann@igpm.rwth-aachen.de,mueller@igpm.rwth-aachen.de \\ Philippe Helluy, Hélène Mathis \\ IRMA, Université de Strasbourg \\ 7 rue René Descartes, 67084 Strasbourg, France. \\ E-mail: helluy@math.unistra.fr, mathis@math.unistra.fr
}

\begin{abstract}
In this paper, we propose a numerical method to model the dynamical behavior of a spherical bubble of vapor and air inside water. The air is assumed to be miscible with the vapor. Each phase is described by a stiffened gas law and the mixture pressure law is recovered by an entropy maximization process. We use an adaptive finite volume solver in order to solve the Euler equations. Numerical experiments are presented that validate our approach.
\end{abstract}

\section{Introduction}

In the present work, we focus on the modeling of the collapse and rebound of a single gas-filled spherical bubble in a liquid. This topic has been subject of numerous numerical and experimental investigations, see for instance [5], [3]. Here, we consider three-phase mixtures, i.e., a bubble of vapor and air is immersed in liquid water. Since the air is a non-condensable gas, it will act as an elastic material in the rebound. Stiffened gas laws are used to depict the thermodynamical behavior of each phase. The mixture pressure law is obtained from an entropy maximization procedure [2], where we assume thermodynamical equilibrium in the mixture. This procedure allows us to compute the mass fraction of vapor in the spirit of [1]. Once it is done, all the other quantities can be computed. In particular, the whole procedure determines the mixture pressure as a function of the internal energy, the specific volume and the mass fraction of air. Note that it defines in a unique manner the mass and volume fractions of all the three phases. 
The outline is as follows. In Section 2, we describe the mixture pressure law construction. This pressure is then coupled with the fluid model introduced in Section 3. Finally some numerical results are presented in Section 4 .

\section{Construction of the mixture pressure law}

We consider a mixture of three phases (1), (2) and (3) representing the vapor, the liquid and the non-condensable gas (air), respectively. The liquid is not miscible with the two others, while the vapor and the gas are miscible. We only admit phase transition between vapor (1) and liquid (2).

Each phase obeys a stiffened gas Equation Of State (EOS), where the entropy function is defined as

$$
s_{i}=C_{i} \ln \left(\left(e_{i}-Q_{i}-\pi_{i} \tau_{i}\right) \tau_{i}^{\gamma_{i}-1}\right)+s_{i}^{0}, \text { for } i=1,2,3 .
$$

In this formula, $C_{i}$ is the specific heat at constant volume, $Q_{i}$ is the heat of formation, $\pi_{i}$ is the reference pressure and $s_{i}^{0}$ the reference entropy. The specific energy is noted $e_{i}$ and the specific volume $\tau_{i}$ is $1 / \rho_{i}$.

Let us recall some basic computations related to the stiffened gas EOS. The temperature $T_{i}$ and the pressure $p_{i}$ are given by

$$
\frac{1}{T_{i}}=\frac{\partial s_{i}}{\partial e_{i}}, \quad \frac{p_{i}}{T_{i}}=\frac{\partial s_{i}}{\partial \tau_{i}} .
$$

Inserting (2.1) this implies the following relations

$$
\begin{aligned}
C_{i} T_{i} & =e_{i}-Q_{i}-\pi_{i} \tau_{i}, \\
p_{i}+\pi_{i} & =\left(\gamma_{i}-1\right) \rho_{i} C_{i} T_{i} .
\end{aligned}
$$

The chemical potential $\mu_{i}=e_{i}+p_{i} \tau_{i}-T_{i} s_{i}$ thus is

$$
\mu_{i} Q_{i}+\gamma_{i} C_{i} T-T\left(C_{i} \ln \left(C_{i} T \tau_{i}^{\gamma_{i}-1}\right)+s_{0}^{i}\right) .
$$

We now consider a mixture of the three phases. We denote by $V_{i}$ the volume, $M_{i}$ the mass and $E_{i}$ the energy of each phase. We wish to compute the pressure law for a mixture of volume $V$, mass $M$ and energy $E$. In other words, we want to compute the pressure from the knowledge of $\tau=V / M, e=E / M$ and the mass fraction $\varphi_{3}=M_{3} / M$ of the non-condensable gas, i.e., $p=p\left(\tau, e, \varphi_{3}\right)$. We introduce the volume fraction $\alpha_{i}=V_{i} / V$, the mass fraction $\varphi_{i}=M_{i} / M$ and the energy fraction $\zeta_{i}=E_{i} / E$ of phase $(i)$. With the fractions, we can express the partial intensive quantities from the global ones

$$
\tau_{i}=\frac{\alpha_{i}}{\varphi_{i}} \tau, \quad e_{i}=\frac{\zeta_{i}}{\varphi_{i}} e
$$


Because phases (1) and (3) are miscible and phase (3) is immiscible with the other phases, the volumes are subject to the constraints

$$
V_{1}=V_{3}, \quad V_{1}+V_{2}=V .
$$

The conservation of mass and energy read

$$
M=M_{1}+M_{2}+M_{3}, \quad E=E_{1}+E_{2}+E_{3} .
$$

From these constraints, we deduce

$$
\begin{aligned}
& \alpha_{1}=\alpha_{3}, \quad \alpha_{1}+\alpha_{2}=1, \\
& \varphi_{1}+\varphi_{2}+\varphi_{3}=1, \quad \zeta_{1}+\zeta_{2}+\zeta_{3}=1 \text {. }
\end{aligned}
$$

At this point, the pressure would depend on $\tau, e, \varphi_{3}$ but also on $\alpha_{1}, \varphi_{1}, \zeta_{1}, \zeta_{2}$. We have to eliminate the four supplementary fractions. The computation proceeds in three steps, which consist in setting the temperature, the pressure and the chemical equilibrium.

Temperature equilibrium. Setting $T=T_{1}=T_{2}=T_{3}$, we eliminate the energy fractions $\left(\zeta_{1}, \zeta_{2}\right)$. Using relations $(2.2)$ and $(2.4)$, we obtain

$$
\sum_{i=1}^{3} \varphi_{i} C_{i} T=e-\sum_{i=1}^{3} \varphi_{i} Q_{i}-\sum_{i=1}^{3} \alpha_{i} \pi_{i} \tau
$$

We then define

$$
C=\sum_{i=1}^{3} \varphi_{i} C_{i}, Q=\sum_{i=1}^{3} \varphi_{i} Q_{i}, \pi=\sum_{i=1}^{3} \alpha_{i} \pi_{i}
$$

in such a way that $C T=e-Q-\pi \tau$. Analogously, we derive

$$
p+\pi=\sum_{i=1}^{3} \alpha_{i}\left(\gamma_{i}-1\right) \frac{\varphi_{i}}{\alpha_{i}} \rho C_{i} T
$$

Next we introduce

$$
\gamma=\frac{\sum_{i=1}^{3} \varphi_{i} \gamma_{i} C_{i}}{\sum_{i=1}^{3} \varphi_{i} C_{i}}
$$

in such a way that

$$
p+\pi=(\gamma-1) \rho C T \text {. }
$$

We observe that assuming the temperature equilibrium leads again to a stiffened gas EOS

$$
p=(\gamma-1) \rho(e-Q)-\gamma \pi
$$

where the mixture parameters are defined by (2.8). This way, the mixture parameters do not depend on the energy fractions. 
Pressure equilibrium. The gas pressures satisfy Dalton's law $\tilde{p}=$ $p_{1}+p_{3}$. The non-miscibility of the gases implies that $p=\tilde{p}=p_{2}=$ $p_{1}+p_{3}$. Using relation (2.10), we get

$$
\begin{aligned}
\left(\left(\gamma_{1}-1\right) \frac{\varphi_{1}}{\alpha_{1}} \frac{C_{1}}{C}+\left(\gamma_{3}-1\right) \frac{\varphi_{3}}{\alpha_{1}} \frac{C_{3}}{C}\right) \rho C T-\pi_{1}-\pi_{3}= \\
\left(\gamma_{2}-1\right) \frac{1-\varphi_{1}-\varphi_{3}}{1-\alpha_{1}} \frac{C_{2}}{C} \rho C T-\pi_{2},
\end{aligned}
$$

where

$$
C T=e-Q-\left(\alpha_{1}\left(\pi_{1}+\pi_{3}-\pi_{1}\right)+\pi_{2}\right) \tau .
$$

If we multiply (2.11) by $\alpha_{1}\left(1-\alpha_{1}\right)$, we obtain a second order equation in $\alpha_{1}$. It is easy to check that this equation admits one single root in $(0,1)$ denoted by $\alpha_{1}\left(\varphi_{1}\right)$.

Chemical equilibrium. From the chemical equilibrium, we compute $\varphi_{1}$. The first step is to express the chemical potential as a function of $\tau, e$ and $\varphi_{1}$, i.e.,

$$
\begin{aligned}
\mu_{i}= & Q_{i}+\frac{C_{i}}{C}(e-Q-\pi \tau) \times \\
& \left(\gamma_{i}-s_{i}^{0}-\ln \left(\frac{C_{i}}{C}(e-Q-\pi \tau)\left(\frac{\alpha_{i}}{\varphi_{i}} \tau\right)^{\gamma_{i}-1}\right)\right),
\end{aligned}
$$

where the mixture parameters $C, Q, \pi$ are given by (2.8). Now we need to determine $\varphi_{1}$ in order to compute the pressure law. Going back to chemistry theory, we apply the following procedure

- if $\mu_{1}\left(1-\varphi_{3}\right)<\mu_{2}\left(1-\varphi_{3}\right)$, the mass fraction of the liquid $\varphi_{2}=0$ corresponds to a stable case, thus $\varphi_{1}=1-\varphi_{3}$, i.e., the mixture contains no liquid but vapor only;

- else if $\mu_{1}(0)>\mu_{2}(0)$, the mass fraction of the liquid $\varphi_{2}=1-\varphi_{3}$ corresponds to a stable case, thus $\varphi_{1}=0$, i.e., the mixture contains no vapor but liquid only;

- else the mixture is necessarily at saturation and we have to compute the mass fraction $\varphi_{1}$ such that $\mu_{1}\left(\varphi_{1}\right)=\mu_{2}\left(\varphi_{1}\right)$. This gives a mass fraction such that $0<\varphi_{1}<1-\varphi_{3}$, i.e., the mixture is at saturation and contains vapor and liquid.

Once the mass fraction $\varphi_{1}$ is computed, it is possible to compute all the other quantities and thus the mixture pressure $p=p\left(\tau, e, \varphi_{3}\right)$ according to $(2.10)$. 


\section{Compressible fluid model}

Once the pressure law is defined, we consider the three-phase mixture as a single inviscid compressible medium described by the density $\rho$, the pressure $p$, the velocity $u$ and the internal energy $e$, all depending on the space variable $x$ and the time $t$. The balances of mass, momentum and energy lead to the Euler equations

$$
\left\{\begin{array}{l}
\partial_{t}(\rho)+\partial_{x}(\rho u)=0, \\
\partial_{t}(\rho u)+\partial_{x}\left(\rho u^{2}+p\right)=0, \\
\partial_{t}(\rho E)+\partial_{x}((E+p) u)=0
\end{array}\right.
$$

where $E=e+u^{2} / 2$ is the total energy. In principle, the amount of air should be evolved by the homogeneous transport equation

$$
\partial_{t} \varphi_{3}+u \partial_{x} \varphi_{3}=0 .
$$

In order to avoid the pressure oscillation phenomenon, described for instance in [6], we consider a uniformly constant fraction of air $\varphi_{3}$. Finally, the system (3.1) is closed by the pressure law $p=p\left(\tau, e, \varphi_{3}\right)$ according to the procedure described in Section 2.

\section{Numerical results}

The first test case consists in a Riemann problem which presents two rarefaction waves moving in opposite directions in water. The value of $\varphi_{3}$ is varying in the range $\left[10^{-9}, 10^{-1}\right]$. The computational domain is $[0,1]$ and the simulation time is $0.2 \mathrm{~ms}$. The computations are performed by an adaptive multiresolution finite volume solver, cf. [4]. The coarse grid consists of $N_{0}=20$ cells and is locally refined by at most $L$ refinement levels. $L$ varies between 5 and 15 and the CFL number is 0.8 . The initial conditions are $\rho_{R}=\rho_{L}=1000 \mathrm{~kg} \cdot \mathrm{m}^{-3}, u_{R}=-u_{L}=100 \mathrm{~m} \cdot \mathrm{s}^{-1}$ and $e_{R}=e_{L}=1.32 \times 10^{5} \mathrm{~J}$ for the right and left state, respectively. The physical parameters are listed in Table 1.

First of all, we observe in Figure 4.1a that the rarefaction waves are smeared for $L=5,7$ whereas the smearing reduces significantly for $L \geq$ 10. In Figure $4.1 \mathrm{~b}$, we note that the density drops significantly in the cavitation zone around $x=0.5 \mathrm{~m}$ with decreasing $\varphi_{3}$. When the amount of air is close to zero, the density is almost zero in the cavitation zone. In particular, the higher the amount of air, the less vapor is in the cavitation zone. Indeed the air occupies the area, which limits the vaporization process as can be observed in Figure 4.1d. Thus from (2.6), it can be deduced that with increasing $\varphi_{3}$, the volume fraction of liquid also increases. Hence, the density is higher in the cavitation zone. Note that 
the initial pressure changes as $\varphi_{3}$ varies, see Figure 4.1c. However, this change in pressure does not affect the conserved quantities. In Figure $4.1 \mathrm{c}$, we observe in the pressure profile discontinuities in the cavitation region when $\varphi_{3}=10^{-9}$. These are probably split waves due to the phase transition across the saturation line.

\begin{tabular}{|c|c|c|c|}
\hline & phase (1) (vapor) & phase (2) (liquid) & phase (3) (air) \\
\hline$\gamma$ & 1.3 & 3 & 1.4 \\
$\pi$ & 0 & $8533 \times 10^{5}$ & 0 \\
$C_{v}$ & 1614.38 & 1400 & 719.28 \\
$Q$ & 1892000 & $-0.1148 \times 10^{7}$ & $-0.26979 \times 10^{6}$ \\
\hline
\end{tabular}

Table 1: Physical parameters.

The second test case is a collapse of a spherical vapor bubble of radius $0.7469 \mathrm{~mm}$. The vapor state is determined by $\rho_{V}=9.57 \times 10^{-4} \mathrm{~kg} . \mathrm{m}^{-3}$, $u_{V}=0 \mathrm{~m} \cdot \mathrm{s}^{-1}$ and $e_{V}=1.90795263 \times 10^{6} \mathrm{~J}$ whereas the ambient liquid state is $\rho_{L}=1000 \mathrm{~kg} . \mathrm{m}^{-3}, u_{L}=0 \mathrm{~m} . \mathrm{s}^{-1}$ and $e_{L}=1.32 \times 10^{5} \mathrm{~J}$, see [5]. Again, the physical parameters are those in Table 1. We can observe in Figure 4.2a that $\varphi_{3}$ has an important effect on the collapse time. The higher the amount of air, the faster the collapse is. This is due to the fact that the pressure increases in the liquid when the amount of air increases but keeping the same conservative variables. When the shock focuses in the center, pressure and temperature increase significantly such that condensation occurs in the bubble center. As the bubble is collapsing, the region of pure vapor, indicated by the isoline $\alpha_{3}=0.99$, reduces. Thus the reflected shock $(r s)$ interacts with the liquid at $t=0.85 \mu \mathrm{s}$ and $x=0.27 \mathrm{~mm}$ and it splits in a transmitted shock $(t s)$ in the liquid and in a reflected shock ( $r r s$ ) moving towards the bubble center. Finally, a reasonable value for $\varphi_{3}$ to investigate bubble oscillations is around $10^{-3}$.

\section{Conclusions}

In this paper we have proposed a cavitation model with a fixed amount of non-condensed gas. Assuming thermodynamical equilibrium in the mixture, we have shown that the mixture pressure law can be computed as a stiffened gas law. This allows us to use a classical finite volume scheme with a relaxation method. The numerical results illustrate a cavitation behavior depending on the amount of air.

In a forthcoming work we will investigate whether the pressure jumps that can be observed in the cavitation region of test case 1, are caused by the saturation line of the mixture. 


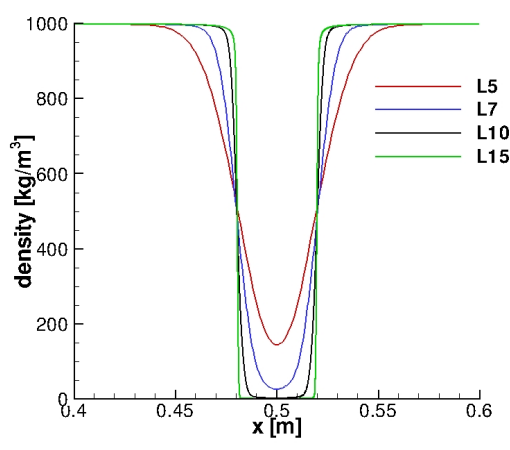

(a)

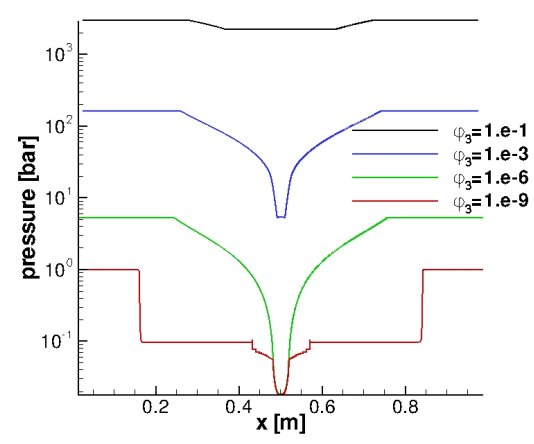

(c)

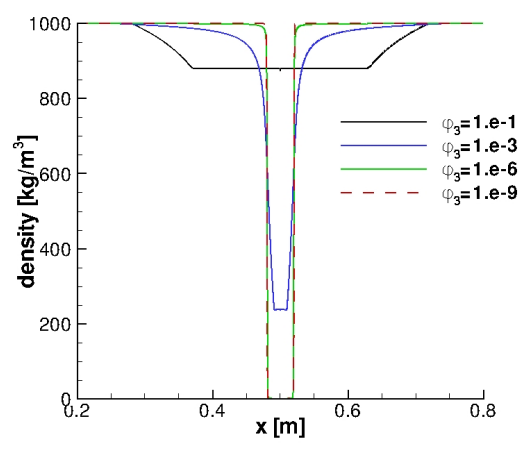

(b)

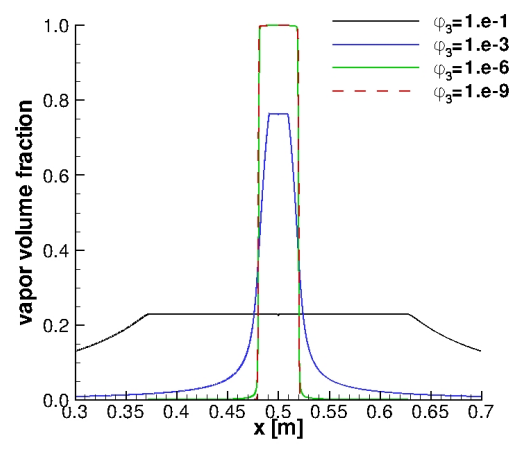

(d)

Figure 4.1: Test case 1: (4.1a) density profiles for $\varphi_{3}=10^{-6}$ and different discretizations. (4.1b) density, (4.1c) pressure, vapor volume fraction for different values of $\varphi_{3}$ and $L=15$.

Another step is to use a non-constant mass fraction of air. Numerical difficulties may arise. In particular, pressure oscillations can appear at the interface between vapor and water, like those observed in many works about two-phase flows, see for instance [6] and [1].

\section{References}

[1] T. Barberon and P. Helluy, Finite volume simulation for cavitating flows. Comput. Fluids 34 (2005), no. 7, 832-858. 


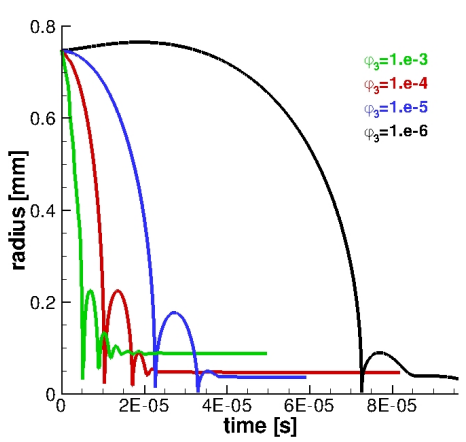

(a)

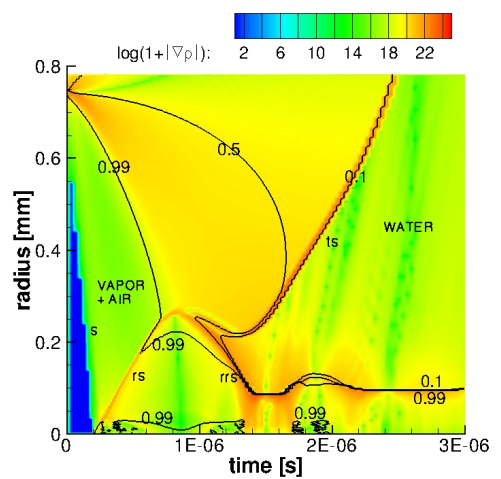

(b)

Figure 4.2: Test case 2: (4.2a) isoline $\alpha_{3}=0.5$ for computations with $\varphi_{3}=10^{-3}, 10^{-4}, 10^{-5}$ and $10^{-6}$. (4.2b) density gradient magnitude and isolines of $\alpha_{3}$ in case of $\varphi_{3}=10^{-2}$.

[2] H. B. Callen, Thermodynamics and an introduction to thermostatistics. Wiley, New-York, 1985.

[3] W. Lauterborn and T. Kurz, Physics of bubble oscillations. Rep. Prog. Phys. 73 (2010) 106501.

[4] S. Müller, Adaptive multiscale schemes for conservation laws. In Lecture Notes in Computational Science and Engineering, volume 27. Springer, 2003.

[5] S. Müller, M. Bachmann, D. Kröninger, T. Kurz, P. Helluy, Comparison and validation of compressible flow simulations of laserinduced cavitation bubbles. Comput. Fluids 38 (2009), no. 9, 18501862.

[6] R. Saurel and R. Abgrall, M. Choulli, A simple method for compressible multifluid flows. SIAM J. Sci. Comput. 21 (1999), no. 3, $1115-1145$. 